\title{
The Research to Service Robot Localization of Inversion GPS in the Spherical Coordinates
}

\author{
Zhibing Deng ${ }^{1, a}$, Siyong $\mathrm{Chu}^{1, \mathrm{~b}}$ and Linli $\mathrm{Tu}^{1, \mathrm{c}}$ \\ ${ }^{1}$ School of Automation, Wuhan University of Technology, Wuhan 430000, China; \\ a835759486@qq.com, b1035613446@qq.com, '1152203676@qq.com
}

Keywords: IGPS, spherical coordinates, ultrasonic.

\begin{abstract}
This paper announces an algorithm to localize service robot in the spherical coordinates and presents a method to implement ultrasonic localization of IGPS (inversion GPS) in the spherical coordinates. In addition, the algorithm is justified after a simulation.
\end{abstract}

\section{Introduction}

With the development of the service robot technology, better daily services are provided with a lot of key issues on service robot solved. The localization of service robot, that the robot needs to know where it is before executing following orders, is still a key issue. Considering some climbing robots are invented and manufactured, as predicted, the altitude parameter will play an important role in the localization technology for now. This paper presents an IGPS algorithm in the spherical coordinates, well solved the problem of service robot localization.

\section{Organization of the Text}

IGPS Algorithm in the Spherical Coordinates. In this section, a localization algorithm in the spherical coordinates is presented. Its principle shows in Fig. $1 . \mathrm{N}$ is for any undetermined nodes with ultrasonic launcher in sensor networks. $S_{1}, S_{2}$ and $S_{3}$ are for three beacon nodes with ultrasonic receiving sensor. After calculating distances between $N$ to $S_{1}, S_{2}$ and $S_{3}$ through ultrasonic, the position where $\mathrm{N}$ is in the spherical coordinates can be written as $(\rho, \theta, \varphi)$.

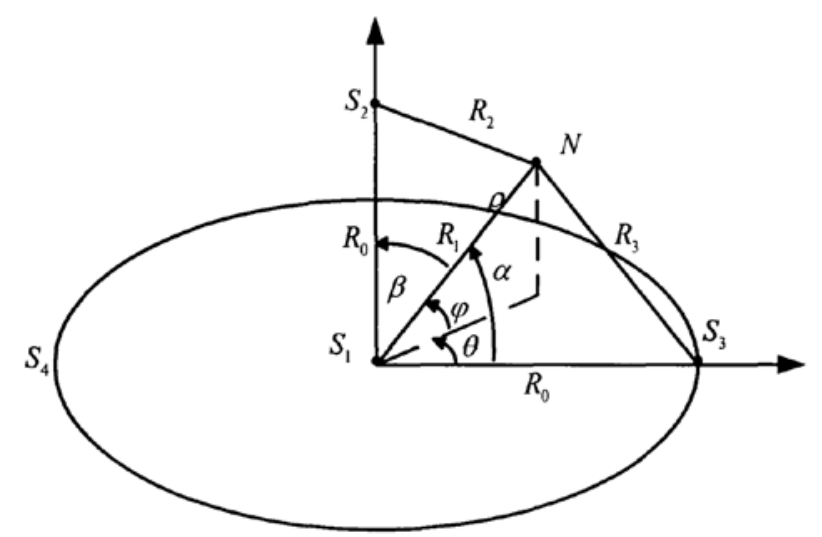

Fig. 1 Nodes denoted in the spherical coordinates

In this coordinate, $\rho$ is for the slant distance which means how far $\mathrm{N}$ is away from $\mathrm{S}_{1}, \theta$ is for the azimuth angle and $\varphi$ is for the elevation angle.

In the spherical coordinates, as the radius of the sphere, $\mathrm{R}_{0}$ is for the max distance to enable receiving signals. This algorithm requires 4 beacon nodes, one at the center of sphere as $S_{1}$, another at the intersection of the plumb line and the sphere as $S_{2}$, another at the north pole of the sphere as $S_{3}$ and the last at the south pole of the sphere as $\mathrm{S}_{4}$.

To those nodes at the northern hemisphere, in the localization system, 3 parameters of propagation delay, from $\mathrm{N}$ to $\mathrm{S}_{1}, \mathrm{~S}_{2}$ and $\mathrm{S}_{3}$, are available. The distance from $\mathrm{N}$ to these receiving terminals is equal to the product of their propagation delay and the signal speed. 


$$
R_{i}=c \tau_{i} \quad(\mathrm{i}=1,2,3)
$$

In this equation, $c$ is for the speed of sound waves propagating in the air, and $\tau_{i}$ is for the propagation delay that sound waves propagate from $\mathrm{N}$ to these receiving terminals.

Then the coordinate of $\mathrm{N}$ can be deduced mathematically shown as $(\rho, \theta, \varphi)$.

$$
\begin{aligned}
& \rho=R_{i} \\
& \varphi=\arcsin \frac{R_{0}{ }^{2}+R_{1}{ }^{2}-R_{2}{ }^{2}}{2 R_{1} R_{0}} \\
& \theta=\arccos \frac{R_{0}{ }^{2}+R_{1}{ }^{2}-R_{3}{ }^{2}}{2 R_{1} R_{0} \cos \varphi}
\end{aligned}
$$

To the other nodes at the southern hemisphere, the calculation method is similar.

The characteristics of this algorithm are as follows:

1. All the beacon nodes are placed at the special points of spherical coordinates. The node coordinate is based on the spherical coordinates. There are less necessary beacon nodes and less calculated quantity.

2. Place ultrasonic launcher at the undetermined node and ultrasonic receivers at beacon nodes.

3. Calculate the distances between $\mathrm{N}$ and these beacon nodes, and transmit them to the data processing control center. Finally, the control center calculates the specific location.

The principle of GPS is one-way ranging, signals transmitting from several satellites to users. It involves two clocks, one of the satellite and the other of the receiver. Once a receiver gets the signal, the satellite clock will be received at the same time. By comparing two clocks, you can get how far it is from satellite to the user by multiplying the difference and light velocity.

The mentioned ultrasonic localization algorithm in the spherical coordinates above, whose transmitting direction is inversion against GPS that beacon nodes receive signals while the undetermined node transmits signal, is called IGPS algorithm in the spherical coordinates.

Distance Measurement Method By Ultrasonic. After determined the localization algorithm, the localization coordinate depends on the estimation of distance between beacon nodes and the undetermined one. The velocity of sound waves in the air can be seen as a constant value $(340 \mathrm{~m} / \mathrm{s})$. The distance between beacon nodes and the undetermined one can be transformed into the measurement of the propagation delay.

This paper estimates the propagation delay by matching filtering results of PRBS (pseudo random binary sequence) signals. The undetermined node transmits PRBS signals and beacon nodes receive and process them. The propagation delay is at the peak position in matching.

The spread-spectrum ultrasonic launcher, carried by the undetermined node, is shown in Fig. 2.The ultrasonic receiver, carried by beacon nodes, is shown in Fig. 3.

The PRBS can be generated by Matlab in advance and saved in the memorizer of the spread-spectrum ultrasonic launcher. A ultrasonic transducer can be seen as a band-pass filter, which makes it happen that replace sine wave into square wave as the carrier and leads to simplification of hard-ware on nodes and lower expense as well. The modulation of the system is BASK, to transmit ultrasonic only when pseudo random code is high level. In order to launch the ultrasonic of the undetermined node, strong voltage signals, which can drive ultrasonic transmitting transducer, are transformed by PRBS and weak voltage signals by level conversion and power amplification. The main function of receiving circuits at beacon nodes is to amplify received weak signals and send to acquisition circuits. 


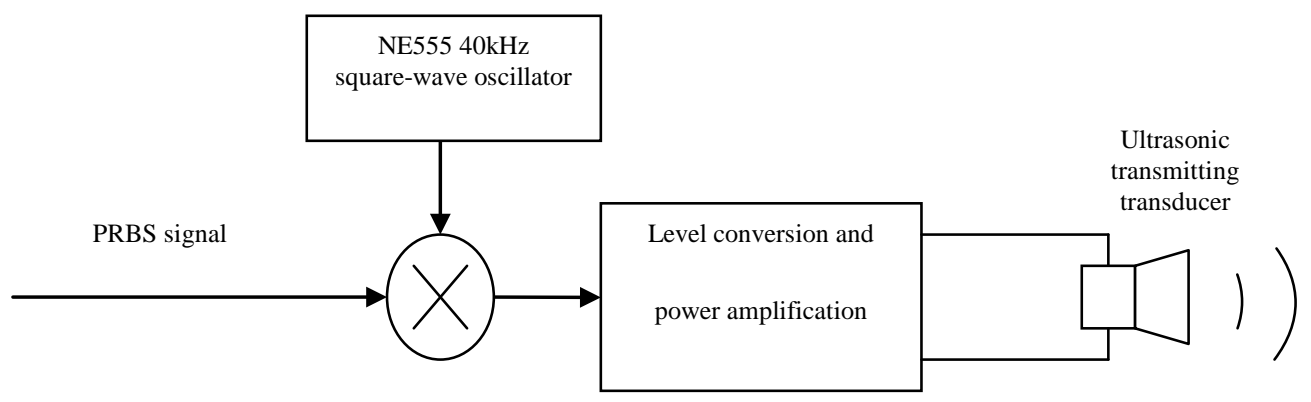

Fig. 2 The spread-spectrum ultrasonic launcher carried by the undetermined node

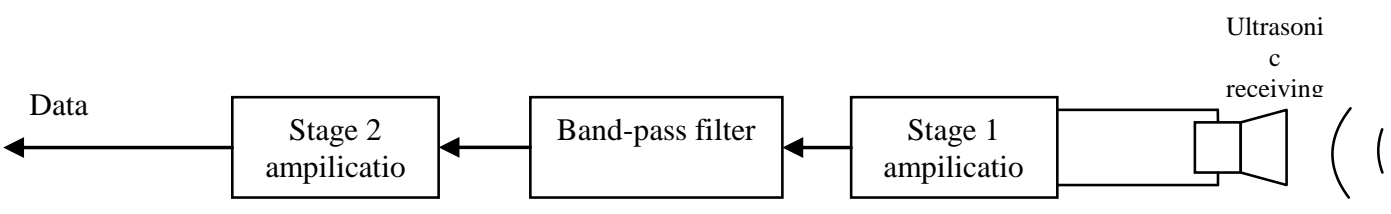

Fig. 3 The ultrasonic receiver carried by beacon nodes

Simulation. In order to justify IGPS Algorithm in the Spherical Coordinates, there are the results of simulation. In this simulation, 127-bit M sequence is as PRBS, which characteristic polynomial is $F(x)=1+x+x^{7}$. The center frequency of the transducer is $40 \mathrm{kHz}$ while sampling frequency is $200 \mathrm{kHz}$ as $f$. FIR filter produced by Matlab is used here as band-pass filter. $\mathrm{R}_{0}$ is set to $15 \mathrm{~m}$. According to analysis above, the position of the peak is corresponding to delay points of two signals. Formula 1can be rewritten if delay point is set to $N_{i}$.

$$
R_{i}=c \frac{N_{i}}{f} \quad(\mathrm{i}=1,2,3)
$$

The results of matching band-pass in beacon nodes are shown as follows.

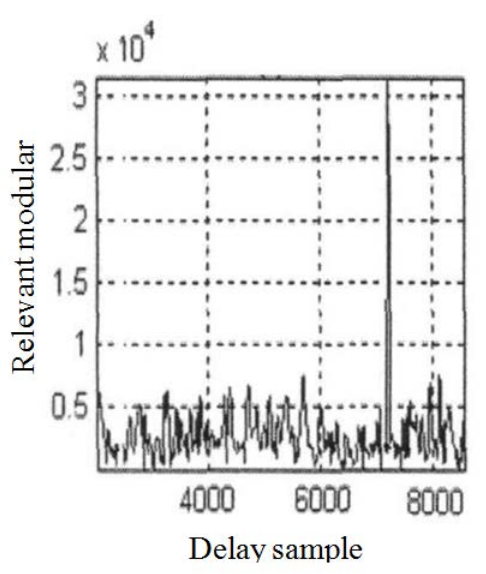

(a)matching with $\mathrm{S}_{1}$

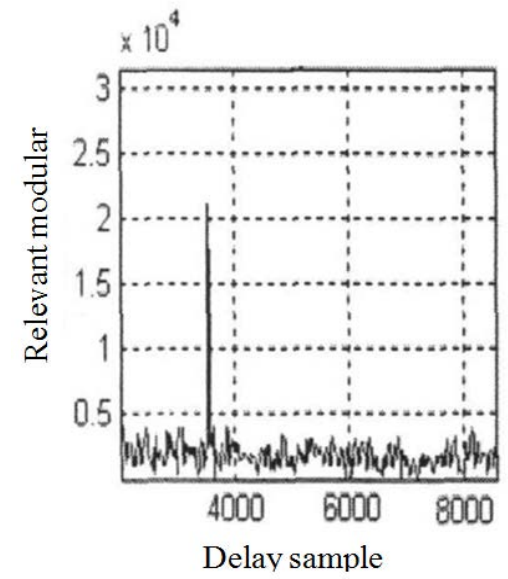

(b)matching with $\mathrm{S}_{2}$

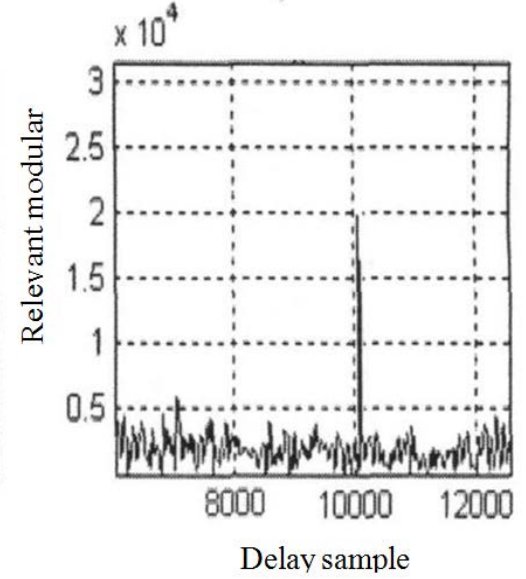

(c)matching with $\mathrm{S}_{3}$

Fig. 4 The results matching with $S_{1}, S_{2}$ and $S_{3}$

Find the position of the peak in Fig. 4, and then $N_{1}=7359, N_{2}=3575, N_{3}=10588$.Plug $N_{i}, f=200 \mathrm{kHz}$ and $c=340 \mathrm{~m} / \mathrm{s}$ into Formula 5, and then $R_{1}=12.51 \mathrm{~m}, R_{2}=6.08 \mathrm{~m}, R_{3}=18.00 \mathrm{~m}$.

According to Formula 2,3 and 4, the undetermined node can be expressed as $\left(12.5103,67.25^{\circ}, 66.65^{\circ}\right)$.

\section{Summary}

This paper announced an IGPS algorithm in the spherical coordinates, that solves the problem to measuring the altitude parameter of the service robot. At the following part, a distance measurement method by ultrasonic is provided and put forward a method to measure distance by transmitting PRBS. At last, a simulation is given to justify the IGPS algorithm. 


\section{References}

[1] Dean S. Oliver, Yan Chen. Recent progress on reservoir history matching: a review[J]. Computational Geosciences, 2011.

[2] Wang C, Liu K, Xiao N. A Range Free Localization Algorithm Based on Restricted-Area for Wireless Sensor Networks[C]// Computing in the Global Information Technology, International Multi-Conference on. IEEE, 2008:97-101.

[3] Yihua H, Zongyuan L, Guojun L. An Improved Bayesian-Based RFID Indoor Location Algorithm[C]// Computer Science and Software Engineering, International Conference on. IEEE, 2008:511-514. 